\title{
Working Process Modeling and Performance Simulation of Half-Crank Internal Combustion Pump
}

\author{
Yuqin Jiao", a, Lei Fu", b, Qinghai Zhao 2, c and Hongxin Zhang ${ }^{1, d}$ \\ ${ }^{1}$ College of Electro mechanic Engineering, Qingdao University, Qindao 266071, China \\ ${ }^{2}$ Power Integration and Energy Storage Systems Engineering Technology Center, Qingdao University, \\ Qingdao 266071, China \\ ajiaoyuqin_qdu@126.com, bfl_qdu@foxmail.com, czqhbit@163.com, dqduzhx@126.com
}

Keywords: Half-Crank internal combustion pump, Dynamic model, Performance simulation, Fuel consumption.

\begin{abstract}
Half-Crank Internal Combustion Pump (HICP) is a new type agricultural power machine converting indicated work into pressure energy, whose crankshaft comprises only half a crank, reciprocating inertial forces of cylinders can entirely counteract each other, the structure is simple and compact, the route of energy transferring is shorter, the technologies based on are mature also. Considering the nonlinear factors including friction, coupling of fluid-solid-gas, etc., and the collaborative mechanism between many disciplines, the dynamic model of HICP is constructed to solve the transient movement and force. Facing the whole working process simulation of HICP, the stationary point iteration method through adjusting the output pressure to arrive at steady working state, is designed, so, the time course of every movement and force during one working cycle can be gotten for the analysis of power and economic performance. The evaluating index system of the performance of HICP was provided also. Through simulation, when throttle changes between $30 \% \sim 100 \%$ and rotational speed changes between $900 \mathrm{r} / \mathrm{min} \sim 2300 \mathrm{r} / \mathrm{min}$, the output pressure and fuel consumption changed within $1.923 \mathrm{Mpa} \sim 10.554 \mathrm{Mpa}$ and $220.0333 \mathrm{~g} /(\mathrm{kW} \cdot \mathrm{h}) \sim 365.9479 \mathrm{~g} /(\mathrm{kW} \cdot \mathrm{h})$ respectively. The pressure is slightly influenced by rotational speed. Fuel consumption shows a middle concave shape and the best point is around the rotational speed of $1300 \mathrm{r} / \mathrm{min}$, and the fuel throttle of $70 \%$. The nonlinear dynamic model and simulation method effectively realized the working process and performance simulation of HICP, of course, is suitable for many other machines also, and the simulation precision is decided by the boundary conditions.
\end{abstract}

\section{Introduction}

Pumps as agricultural machinery in the hydraulic seeding, irrigation and drainage, deinsectization, and other aspects have important applications. When the high pressure required, we mainly use motor driven multi-stage pump system or engine drive plunger pump system. The latter is mainly used for power access to inconvenient field or flexible mobile operations. It is a power plant that converts fuel combustion heat energy into fluid pressure energy. Although the technology is mature, widely used, but the traditional internal combustion engine and piston pumps as two separate units is difficult to achieve optimal matching. And because of the long transmission route of the whole system, the traditional internal combustion engine - piston pump system has the disadvantages of heavy structure, low efficiency, high production cost and poor economic performance ${ }^{[1-2]}$. The power plants with similar functions also includes Hydraulic Free Piston Engine (HFPE) and Hydraulic Confined Piston Engine (HCPE). They can directly convert the indicator work into fluid pressure energy, and the efficiency is high, but there are many problems in HFPE, such as unstable work, complex control, and more technical bottlenecks, so it has not been available to shape products. In the study of HFPE dynamics, Zhao Zhenfeng, Wu Wei, Wei Chao successfully established the HFPE nonlinear dynamic model and completed the experimental study ${ }^{[7-10]}$. For HCPE, the literature [11-15] emphasizes the advantages of good technical inheritance, good performance and other characteristics, but from the structural principle of its comparison with the traditional internal combustion engine, it is not difficult 
to find that the HCPE body is high, the vibration is violent, so it is difficult to popularize. Based on the structure and function of the traditional internal combustion engine driven piston pump system, HFPE and HCPE, this paper presents the structure principle of Half-crank Internal Combustion Pump. The working process is the same as the existing internal combustion fluid machinery, involving complex liquid - solid - gas coupling, combustion, system dynamics, fluid mechanics and other disciplines of the coordination mechanism. This paper will study the nonlinear working process model of HICP and its performance simulation method, which will provide the basis for the future product design optimization and performance evaluation.

\section{HICP dynamics modeling}

\subsection{HICP structure principle}

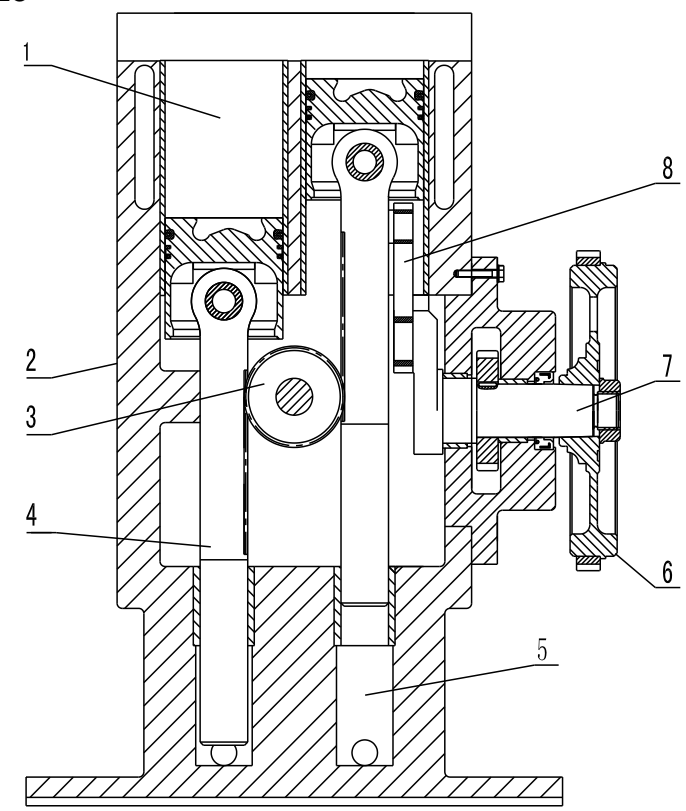

1- Power cavity; 2- Organism; 3- Linkage gear; 4- Piston-plunger assembly; 5- Pump cavity; 6- Free wheel; 7- Half crankshaft; 8- connecting rod

Fig. 1 Structural principle schematic of HICP

As shown in Figure 1, the principle of the HICP is that the crankshaft has only one crank and a connecting rod journal, it doesn't run through the whole body like the crankshaft of the traditional internal combustion engine, which will greatly reduce the cost of production. The piston-plunger assembly of the two cylinders through the linkage gear to achieve synchronous reverse movement, the reciprocating inertia force is completely canceled and the complex defects of the HFPE and HCPE reciprocating inertia force balance structure is completely overcome. HICP power chamber is consistent with the traditional internal combustion engine. When the piston plunger assembly moves upward, the low pressure fluid enters the pump cavity through the flow distribution system, and outputs the high pressure fluid to the outside when moving downwards. The connecting rod and the crank flywheel group are the mechanism which can make the reciprocating movement of the piston-plunger and the rotary motion of the crankshaft mutual transform. It is also used in the HICP working time, the accessory system drive, the piston motion limit, etc. 


\subsection{Nonlinear dynamic model}

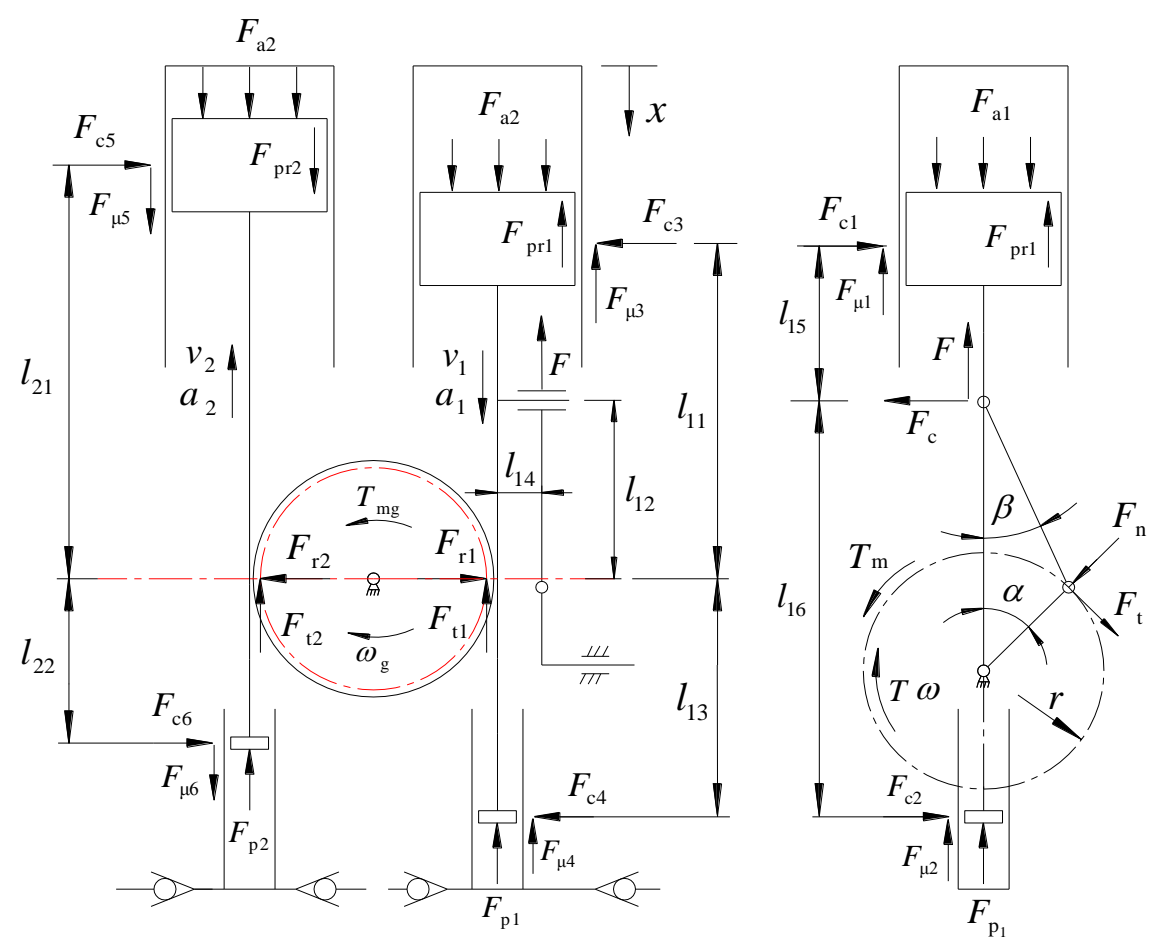

Fig. 2 Dynamics analysis diagram of HICP

Dynamic analysis of HICP as shown in Figure 2, $\alpha$ is the crank angle, $\operatorname{rad} ; \beta$ is a connecting rod angle, $\mathrm{rad} ; v_{1}, v_{2}$, is the 1,2 cylinder piston speed, $\mathrm{m} / \mathrm{s} ; a_{1}, a_{2}$ is the 1,2 cylinder piston acceleration, $\mathrm{m} / \mathrm{s}^{2} ; r$ is the crank radius, $\mathrm{m} ; \omega$ is the crankshaft angular velocity, $\mathrm{rad} / \mathrm{s} ; \omega_{\mathrm{g}}$ is gear angular velocity, $\mathrm{rad} / \mathrm{s} ; F_{a 1}, F_{a 2}$ is 1,2 cylinder gas piston force, $\mathrm{N} ; F$ is the connecting rod piston force along the $\mathrm{x}$ axis, $\mathrm{N} ; F_{c}$ is the connecting rod piston and plunger Assembly of lateral force, $\mathrm{N} ; F_{t}$ is the tangential force for the connecting rod to the crank, $\mathrm{N} ; F_{n}$ is the normal force of the connecting rod to the crank, N; $T$ is a connecting rod on the initiative of the crankshaft torque, $\mathrm{N} \bullet \mathrm{m} ; T_{m}$ is subsidiaries driving torque, $\mathrm{N} \bullet$ $\mathrm{m}$, by the method of reverse drag; $T_{m g}$ is gears friction torque, $\mathrm{N} \bullet \mathrm{m} ; \omega_{\mathrm{g}}$ is gear angular velocity, $\mathrm{rad} / \mathrm{s}$; $F_{c l}$ is $\mathrm{F}_{\mathrm{c}}$ lead the lateral force of the piston on the cylinder, $\mathrm{N} ; F_{\mu l}$ is $F_{c l}$ caused friction between piston and cylinder, $\mathrm{N} ; F_{c 2}$ is $F_{c}$ lead the side force of the plunger bushing, $\mathrm{N} ; F_{\mu 2}$ is $F_{c 2}$ caused piston friction with the Guide bushing, $\mathrm{N} ; F_{r 1}, F_{r 2}$ is linkage gear radial force, $\mathrm{N} ; F_{t 1}, F_{t 2}$, is linkage gear-cutting force, $\mathrm{N} ; F_{c 3}, F_{c 5}$ is lateral force of piston to cylinder liner caused by radial force of linkage gear, $\mathrm{N} ; F_{\mu 3}, F_{\mu 5}$ is the friction force between the piston and the cylinder caused by the radial force of the gear, $\mathrm{N} ; F_{c 4}$, $F_{c \sigma}$ is lateral force caused by the radial force of the linkage gear on the guide sleeve, $\mathrm{N} ; F_{\mu 4}, F_{\mu 6}$ is the friction force between the plunger and the guide sleeve caused by the radial force of the linkage gear, $\mathrm{N} ; F_{p 1}, F_{p 2}$ is the force of the fluid on the plunger assembly, $\mathrm{N} ; F_{p r 1}$, and $F_{p r 2}$ is the friction between the piston ring and the cylinder wall, $\mathrm{N} ; l_{11}$ is the distance between the right cylinder piston pin axis and the linkage gear shaft, $\mathrm{m} ; L_{12}$ is the distance between the connecting rod and the gear axis, $\mathrm{m} ; L_{13}$ is distance from the right cylinder gear shaft to the middle section of the plunger, $\mathrm{m} ; L_{14}$ is the distance between the connecting rod and the right cylinder piston-piston assembly axis, $\mathrm{m} ; L_{15}$ is the distance between the right cylinder piston pin axis and the connecting rod, $\mathrm{m} ; L_{16}$ is the distance from the axis of the connecting rod to the right cylinder, $\mathrm{m} ; L_{21}$ is the distance between the piston pin axis of the left cylinder and the axis of the linkage gear, $\mathrm{m} ; l_{22}$ is the distance between the left cylinder gear shaft and the middle section of the plunger, $\mathrm{m}$. The right cylinder is the first cylinder, the left cylinder is the second cylinder. When modeling the HICP working process, the crank link portion has a similarity to the $\mathrm{HCPE}^{[13-17]}$. Due to the adoption of the rotary sleeve type flow distribution system, the mathematical model of the system is simplified, although the main structure is complex (Limited space no longer repeat). Nonlinear mathematical model of liquid-solid-gas coupling in HICP working process mainly includes four equations: the dynamic balance of the crankshaft flywheel group, the 
dynamic balance of the right cylinder piston-plunger assembly, the dynamic balance of the linkage gear, and the dynamic balance of left cylinder piston-plunger assembly.

$$
\left\{\begin{array}{c}
T-T_{\mathrm{m}}-I_{0} a_{j}=0 \\
F_{\mathrm{a} 1}+F+F_{\mathrm{j} 1}-\operatorname{sign}\left(V_{1}\right) \sqrt{F_{\mathrm{\mu} 1}^{2}+F_{\mathrm{\mu} 3}^{2}}- \\
\operatorname{sign}\left(V_{1}\right) \sqrt{F_{\mathrm{\mu} 2}^{2}+F_{\mathrm{\mu} 4}^{2}}+F_{\mathrm{p} 1}+F_{\mathrm{t} 1}+F_{\mathrm{pr} 1}=0 \\
F_{\mathrm{t} 2} F_{1}-F_{\mathrm{t1}} F_{1}+T_{\mathrm{mg}}+I_{\mathrm{g}} \dot{\omega}_{\mathrm{g}}=0 \\
F_{\mathrm{a} 2}+F_{\mathrm{j} 2}+F_{\mathrm{\mu} 5}+F_{\mathrm{\mu} 6}+F_{\mathrm{p} 2}+F_{\mathrm{t} 2}+F_{\mathrm{pr} 2}=0
\end{array}\right.
$$

In the formula, $a_{\mathrm{j}}$ is the angular acceleration of the crankshaft; $I_{0}$ is the moment of inertia of the crankshaft flywheel group; $I_{\mathrm{g}}$ is the moment of inertia; $r_{1}$ is the radius of the gear pitch circle; sign () is a sign function.

$T$ is the active torque of the connecting rod to the crankshaft:

$T=\underline{F_{t}} \cdot r$

$F_{\mathrm{t}}=F \sin (\alpha+\beta) / \cos \beta$

The force acting on the piston in the 1and 2 cylinder cavity:

$$
\left\{\begin{array}{l}
F_{\mathrm{a} 1}=\pi D^{2}\left(p_{\mathrm{a} 1}-p_{0}\right) / 4 \\
F_{\mathrm{a} 2}=\pi D^{2}\left(p_{\mathrm{a} 2}-p_{0}\right) / 4
\end{array}\right.
$$

In the formula, $\mathrm{D}$ is the piston diameter; $p_{0}$ is the air pressure on the lower surface of the piston; $p_{\mathrm{a} 1}$, $p_{\mathrm{a} 1}$ is the gas pressure in the first and second cylinders, which is obtained by the thermodynamic simulation of the combustion process ${ }^{[17-18]}$, the second cylinder (left cylinder) is delayed by $540{ }^{\circ} \mathrm{CA}$ (Crankshaft Angle) than the first cylinder (right cylinder).

$F_{\mathrm{j} 1}, F_{\mathrm{j} 2}$ is the first and second cylinder reciprocating inertia force:

$\left\{\begin{array}{l}F_{\mathrm{j} 1}=-m_{\mathrm{j} 1} a_{1} \\ F_{\mathrm{j} 2}=-m_{\mathrm{j} 2} a_{2}\end{array}\right.$

In the formula, $m_{\mathrm{j} 1}, m_{\mathrm{j} 2}$ is the reciprocating mass of the first and second cylinders.

The force of the fluid on the plunger assembly:

$$
\left\{\begin{array}{l}
F_{\mathrm{p} 1}=-\operatorname{sign}\left(v_{1}\right) p_{11} S_{\mathrm{z}} \\
F_{\mathrm{p} 2}=-\operatorname{sign}\left(v_{2}\right) p_{12} S_{\mathrm{z}}
\end{array}\right.
$$

In the formula, $p_{11}, p_{12}$ is the first and second cylinder pump pressure; When the plunger is upward, the input pressure and the symbol are positive, when it goes down, the output pressure and symbol are negative; $S_{\mathrm{z}}$ is the plunger end area, $S_{\mathrm{z}}=\pi d^{2} / 4, d$ is the diameter of the plunger.

Friction between moving parts:

$$
\left\{\begin{array}{l}
F_{\mu 1}=-\operatorname{sign}\left(v_{1}\right)\left|F_{\mathrm{c} 1}\right| \mu_{1} \\
F_{\mu 2}=-\operatorname{sign}\left(v_{2}\right)\left|F_{\mathrm{c} 2}\right| \mu_{2} \\
F_{\mu 3}=\operatorname{sign}\left(v_{1}\right) F_{\mathrm{c} 3} \mu_{1} \\
F_{\mu 4}=\operatorname{sign}\left(v_{1}\right) F_{\mathrm{c} 4} \mu_{2} \\
F_{\mu 5}=-\operatorname{sign}\left(v_{2}\right) F_{\mathrm{c} 5} \mu_{1} \\
F_{\mu 6}=-\operatorname{sign}\left(v_{2}\right) F_{\mathrm{c} 6} \mu_{2}
\end{array}\right.
$$

$\mu_{1}$ is the coefficient of friction between piston and cylinder; $\mu_{2}$ is the coefficient of friction between piston plunger assembly and guide sleeve;

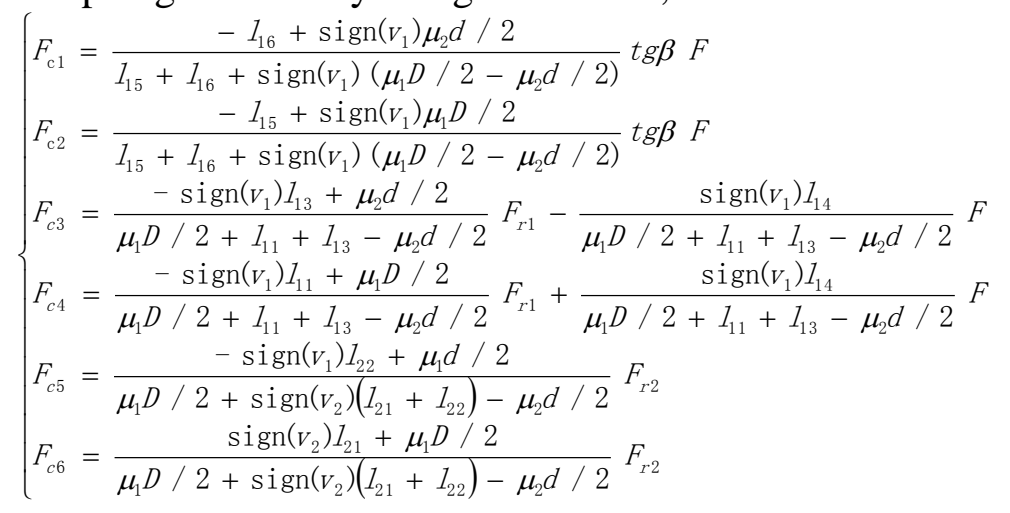


The HICP model has obvious friction and liquid-solid-gas coupling nonlinearity:

(1)Force order nonlinearity; (2)Friction hysteresis nonlinearity; (3) Pump cavity pressure step nonlinearity; (4)Nonlinear variation of gas pressure in dynamic chamber (Obtained by thermodynamic simulation).

\section{Performance simulation method}

\subsection{Performance evaluation index}

The working performance of HICP mainly includes power performance and economic performance. For a stable condition (Fixed speed $\mathrm{n}, \mathrm{r} / \mathrm{min}$, and throttle opening $\alpha, \%$ ), the dynamic performance of HICP can be evaluated by three indexes: the output pressure $p_{\mathrm{c}}(\mathrm{MPa})$, the output flow rate $Q\left(\mathrm{~m}^{3} / \mathrm{h}\right)$ and the operating nonuniformity $\delta(\%)$.Economic performance can be evaluated by three indexes: effective power $P_{\mathrm{e}}(\mathrm{kW})$, effective thermal efficiency $\eta_{\mathrm{et}}(\%)$ and fuel consumption rate $b_{\mathrm{e}}(\mathrm{g} / \mathrm{kW} . \mathrm{h})$. The output pressure has been directly obtained by simulation, $p_{\mathrm{c}}=p^{(k)} / 10^{6} \mathrm{Mpa}$.

Output flow

$Q=60 n n_{\mathrm{p}} \eta_{\mathrm{v}} S_{\mathrm{Z}} S$

In the formula, $n_{\mathrm{p}}$ is the number of plunger, $\eta_{\mathrm{v}}$ is the volumetric efficiency of the system, $\eta_{\mathrm{v}}$ approximate $96 \%$; $S$ is the piston stroke, $\mathrm{m}$.

Running unevenness is the ratio of the maximum angular velocity $\omega_{\max }(\mathrm{rad} / \mathrm{s})$ and the minimum angular velocity $\omega_{\min }(\mathrm{rad} / \mathrm{s})$ to the average angular velocity $\omega_{\mathrm{m}}(\mathrm{rad} / \mathrm{s})$ :

$\delta=\left(\omega_{\max }-\omega_{\min }\right) / \omega_{\mathrm{m}} \times 100 \%$

In the formula, $\omega_{\max }, \omega_{\min }$ have been directly obtained by simulation, $\omega_{\mathrm{m}}=2 \pi n / 60$.

Effective power (HICP output hydraulic power):

$P_{\mathrm{e}}=Q p / 3.6$

Effective thermal efficiency is the ratio of the effective power to the low calorific value of fuel consumed per unit time:

$$
\eta_{e t}=\frac{3.6 \times 10^{3} P_{e}}{B H_{u}}
$$

In the formula, $\mathrm{B}$ is the hourly fuel consumption, $\mathrm{kg} ; H_{\mathrm{u}}$ is the low calorific value of the fuel, the HICP fuel is diesel,

$H_{\mathrm{u}}=42800 \mathrm{~kJ} / \mathrm{kg}$.

Fuel consumption rate refers to the unit effective work of fuel consumption:

$$
b_{e}=\frac{3.6 \times 10^{6}}{\eta_{e t} H_{u}}
$$

\subsection{Simulation method}

In order to obtain the nonlinear dynamical model of HICP of formula (1), we first get $F_{\mathrm{t} 2}$ from equation (4) and then bring $F_{\mathrm{t} 2}$ into equation (3) to get result $F_{\mathrm{t} 1}$, and finally bring $F_{\mathrm{t} 1}$ into equation (1) to get the result $a_{\mathrm{j}}, a_{\mathrm{j}}$ can be used for the overall simulation of the crankshaft angular velocity. At the same time, the solution of the transient condition is completed, and the movement and force of HICP at a given time point are obtained.

The key of the whole simulation process is how to stabilize the HICP as soon as possible under any initial conditions, we can use the fixed point iteration method proposed in [20], the torque control method is discussed in detail, and HICP need to control the output pressure to stabilize at a certain point of work, the specific steps are as follows.

For the steady state (fixed point) with a speed of $n_{0}$ and a throttle opening of $\alpha_{0}$, crankshaft angular velocity:

$\omega_{0}=2 i \pi n_{0} /(60 i)$

In the formula, $i$ is the stroke factor, when the HICP power chamber has 2 working strokes $i=1$, and when the HICP power chamber has 4 working strokes $i=2$, i cannot be omitted, because the simulation 
cycle and convergence criteria are based on 1 working cycles. This paper analyzes the 4 stroke engine, so $\mathrm{i}=2$.

We can take an initial angular velocity $\omega^{(0)}\left(\omega_{0}\right)$ and output pressure $p^{(0)}$ to start the simulation calculation. After a working cycle, the crankshaft angular velocity becomes $\omega^{(1)}$. Adjust the output pressure to:

$$
p^{(1)}=p^{(0)}+\Delta p^{(1)}
$$

In the formula, The output pressure increment $\Delta p^{(1)}$

$\Delta p^{(1)}=c I_{0}\left\{\left[\omega^{(1)}\right]^{2}-\omega_{0}^{2}\right\} /\left(2 i n_{\mathrm{p}} S_{Z} S\right)$

In the formula, $c$ is the magnification factor. The purpose of the above adjustment is to make the next working cycle output hydraulic pressure increment is c times of the kinetic energy increment of the crankshaft flywheel group, according to the experience of $c=2$, we can obtain a satisfactory convergence rate.

In the simulation, the relationship between the $\mathrm{K}$ working cycle and the output pressure of the $\mathrm{k}-1$ working cycles is

$$
p^{(k)}=p^{(k-1)}+\Delta p^{(k)}
$$

In the formula, The output pressure increment $\Delta \mathrm{p}^{(\mathrm{k})}$ of the $\mathrm{K}$ cycle is

$\Delta p^{(k)}=c I_{0}\left\{\left[\omega^{(k)}\right]^{2}-\omega_{0}^{2}\right\} /\left(2 i n_{\mathrm{p}} S_{\mathrm{z}} S\right)$

In the formula, $\omega^{(k)}$ is the average angular velocity of the $\mathrm{K}$ working cycle. Convergence criteria:

$$
\left|\omega^{(k)}-\omega_{0}\right| \leq \varepsilon \text { 或 }\left|\frac{\omega^{(k)}-\omega_{0}}{\omega_{0}}\right| \leq \varepsilon_{1}
$$

In the formula, $\varepsilon, \varepsilon_{1}$ is the convergence precision, it is a smaller number, preferably less than or equal to 0.01 , which ultimately depends on the accuracy requirements. When the simulation is convergent, the output pressure of stable condition, the time of movement and force in 1 working cycles of each part are obtained directly.

In the design of the HICP prototype, the whole structure of the diesel engine is the prototype of the D6114ZG4B diesel engine. The pump cavity and the linkage gear rack parts are designed by ourselves. The coefficient of friction between the piston and the cylinder liner, the crankshaft and the bearing shell is calibrated by the experimental condition of D6114ZG4B. The coefficient of friction between the piston-plunger assembly and the guide sleeve is based on experience $\mu_{2}=0.06$, Other key parameters are: $r=0.0675 \mathrm{~m}, I_{0}=0.68986 \mathrm{~kg} \cdot \mathrm{m}^{2}, m_{\mathrm{j} 1}=5.019 \mathrm{~kg}, \mu_{1}=0.101, D=0.114 \mathrm{~m}, S=0.135, d=0.021 \mathrm{~m}$, $d_{\mathrm{r}}=0.103 \mathrm{~m}, t_{\mathrm{r}}=0.0025 \mathrm{~m}, I_{\mathrm{g}}=0.00407 \mathrm{~kg} \cdot \mathrm{m}^{2}, m_{\mathrm{j} 2}=5.003 \mathrm{~kg}$. The effective working range of the HICP design prototype speed $\mathrm{n}$ is $900 \mathrm{r} / \mathrm{min} \sim 2300 \mathrm{r} / \mathrm{min}$, The effective working range of the throttle opening $\alpha$ is $30 \% \sim 100 \%$, each $n$ and $\alpha$ form a working point $(n, \alpha)$ of HICP. Using the above model and simulation method, we can simulate the performance index of each condition $(n, \alpha)$.

\subsection{Simulation example}

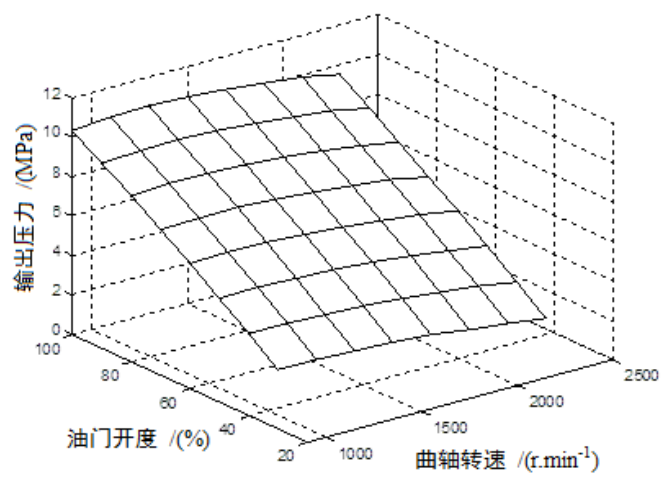

Fig.3 Universal characteristics of output pressure 


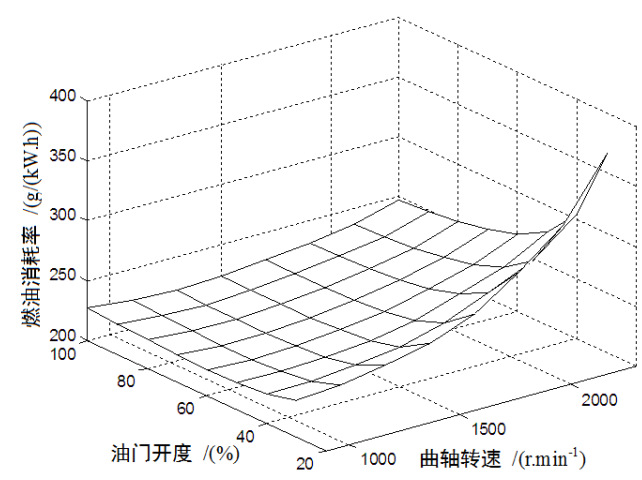

Fig.4 Universal characteristics of fuel consumption

The simulation of output pressure and fuel consumption which are the most two representative indicators of the dynamics and economy of HICP as follows.. The relationship curve about $p$, throttle opening $\alpha$ and crankshaft speed $n$ is shown in Figure 3, the variation range of $p$ is $1.923 \mathrm{Mpa}$ 10.554Mpa. When $\alpha$ was fixed, the change of $\mathrm{N}$ had little effect on $\mathrm{P}$. When $\mathrm{N}$ was fixed, the $\mathrm{P}$ increased linearly with the increase of $\alpha$. The relationship curve about $b_{\mathrm{e}}$, throttle opening $\alpha$ and crankshaft speed $n$ is shown in Figure 4. When $b_{\mathrm{e}}$ is $220.0333 \mathrm{~g} /(\mathrm{kW} \cdot \mathrm{h}) \sim 365.9479 \mathrm{~g} /(\mathrm{kW} \cdot \mathrm{h})$, the overall fuel consumption rate in the concave type, the speed is $1300 \mathrm{r} / \mathrm{min}$, the throttle opening is $70 \%$, fuel economy is the best. And when $\alpha$ is lower and $n$ is high, $b_{\mathrm{e}}$ sharply increases, fuel economy performance deteriorates sharply.

The foregoing models and methods can effectively implement the simulation of the HICP process and performance, and of course apply to many other machines. The accuracy of the simulation depends on the certainty and accuracy of the boundary conditions, such as the value of the air pressure in the crankcase, the friction coefficient of the plunger, and the pressure curve in the dynamic chamber.

\section{Summary}

1) HICP is a new type of agricultural power machinery, which can directly convert part of the indicator power into the fluid pressure energy. It has the advantages of stable operation, compact structure, short transmission path of energy conversion and mature technology.

2) The dynamic model of HICP takes into account the nonlinear factors such as friction, liquid-solid-gas coupling and so on, which can be used to solve the transient motion and force of each structure.

3) By using the fixed point iterative simulation method and adjusting the output pressure of the HICP achieve the stable conditions. Thus, the time history of the motion and force of a component in a working cycle under the working condition is obtained, which is used to analyze the dynamic performance and economic performance.

4) Through simulation, when the HICP output pressure is $1.923 \mathrm{Mpa} 10.554 \mathrm{Mpa}$, fuel consumption rate is $220.0333 \mathrm{~g} /(\mathrm{kW} \cdot \mathrm{h}) \sim 365.9479 \mathrm{~g} /(\mathrm{kW} \cdot \mathrm{h})$, the speed change on the output pressure is not affected, the overall fuel consumption rate in the concave type, the speed is $1300 \mathrm{r} / \mathrm{min}$, the throttle opening is $70 \%$, fuel economy is the best. The model and method studied in this paper can effectively realize the simulation of HICP working process and performance.

\section{Acknowledgements}

This work was supported by the National Natural Science Foundation of China (51575286); Shandong Natural Science Foundation of China (2014ZRB01503).

\section{References}

[1] Jiang Youshan, Zou Guangde, Xu Gang, et al. Matching research of engine and hydraulic pump of full-hydraulic bulldozer [J]. Construction Mechanization, 2009, 30(12): 49-52. 
[2] Li Xiang-sheng, Chang Siqin. Matching Efficiency of Power Source System for Vehicle with Energy Accumulation Hydrostatic Drive [J]. China Journal of Highway and Transport, 2007, 20(1): 118-122.

[3] Mikalsen R, Roskilly A P. A review of free-piston engine history and applications. Applied thermal Engineering 2007; 27:2339-2352.

[4] Mikalsen R, Jones E, Roskilly A P. Predictive piston motion control in a free-piston internal combustion engine. Applied Energy 2010; 87: 1722-1728.

[5] Mikalsen R, Roskilly A P. A computational study of free-piston diesel engine combustion [J]. Applied Energy, 2009, 86(7-8): 1136-1143.

[6] Zhou Sheng, Xu Bing, Yang Huayong, et al. Simulation on dual hydraulic free piston engine [J]. Chinese Journal of Mechanical Engineering, 2005, 41(4): 91-95.

[7] Jin Xiao, Qingfeng Li, Zhen Huang. Motion characteristic of a free piston linear engine. Applied Energy 2010; 87: 1288-1294.

[8] Wei Chao,Wu Wei,Jing Chongbo, Yuan Shihua Movement mechanism of hydraulic free-piston of engine around bottom dead centre [J]. Transactions of the Chinese Society of Agricultural Engineering, 2010, 26(11): 119-123.

[9] Jibin Hu, Wei Wu, Shihua Yuan, et al. Mathematical modelling of a hydraulic free-piston engine considering hydraulic valve dynamics [J]. Energy, 2011, 36(10): 6234-6242.

[10] Zhenfeng Zhao, Fujun Zhang, Ying Huang, et al. An experimental study of the cycle stability of hydraulic free-piston engines [J]. Applied Thermal Engineering, 2013, 54(2): 365-371.

[11] Huo Wei, Zhang Tiezhu, Zhang Hongxin, et al. Best economic working area of three-cylinder internal combustion water pump $[\mathrm{J}]$. Transactions of the Chinese Society of Agricultural Engineering, 2009,25(11): 152-157.

[12] Zhang Tiezhu, Dai Zuoqiang,Zhang Hongxin, et al. Scheme design and simulation result of internal combustion hydraulic system [J]. Chinese Journal of Mechanical Engineering, 2006, 42(5): 169-173.

[13] Yang Daozhai, Zhang Hongxin, Zhang Tiezhu, et al. Working characteristic simulation of single-cylinder hydraulic confined piston engine[J]. Advanced materials rsearch, 2011, 422: 652-656.

[14] Zhao Hong, Zhang Tiezhu, Zhang Hongxin, et al. Dynamic modeling and external characteristic simulation of three-cylinder internal combustion water pump [J]. Transactions of the Chinese Society of Agricultural Engineering, 2009,25(3):114-118.]

[15] Zhang Hongxin, Zhang Tiezhu, Wang Yushun, Zhao Hong, Huo Wei. Dynamic model and simulation of flat valve system of internal combustion water pump [J]. Chinese Journal of Mechanical Engineering, 2005, 18(3): 411-414.

[16]Zhang Hongxin, Zhang Tiezhu, Zhao Hong, et al. Simulation study on working process of single-cylinder axial internal combustion engine [J]. Fluid Machinery, 2007, 35(3): 1-5.

[17] Zhao Hong, Zhang Tiezhu, Zhang Hongxin, et al. Multi-objective Optimization Study of Orifice System of Hydraulic Confined Piston Engine [J]. China Mechanical Engineering, 2008, 19(12): 1479-1482.

[18] Benjamin A. Cantrell, Hai-Wen Ge, Rolf D. Reitz and Christopher J. Rutland. Validation of advanced combustion models applied to two-stage combustion. SAE Technical paper 2009-01-0714.

[19] Rakopoulos C D and Giakoumis E G. Review of thermodynamic diesel engine simulations under Transient operating conditions. SAE Technical paper 2006-01-0884.

[20] Yang Daozhai, Zhang Hongxin, Jiang Yong, et al. Simulation of iteration method with stationary point in working process of internal combustion pump [J]. Transactions of the Chinese Society of Agricultural Engineering, 2011, 27(11): 90-9 